\title{
Prevalencia de depresión postparto en pacientes del Hospital de la Mujer de Aguascalientes
}

\author{
Torres-Cisneros Eduardo Rey*, Barba-Valadez Leonardo Alberto*, \\ Terrones-Saldivar Ma del Carmen**
}

\footnotetext{
Resumen

- La depresión tiene una prevalencia aproximada del 8\% en la - población general. Los trastornos depresivos son más frecuen- tes en la población femenina y han sido asociados a diferen-

- tes eventos vitales, incluído el embarazo, parto y puerperio.

- El objetivo de este trabajo fue conocer la frecuencia de la

- depresión postparto en pacientes atendidas en el Hospital de

- la Mujer de Aguascalientes. Se realizó un estudio descriptivo,

- prospectivo y transversal, se incluyeron, mediante un muestreo

- por conveniencia, pacientes que cursaban el puerperio tardío

- y que fueron citadas en la consulta externa, entre el 15 de oc-

- tubre y 16 de noviembre del 2012. Se utilizó la Escala de De-

- presión Postparto de Edimburgo. Se estudiaron 144 pacientes.

- Encontramos una prevalencia de $21.5 \%$, que representa una

- frecuencia mayor a lo reportado por otros autores. Estos resul-

- tados nos llevan a continuar con esta línea de investigación. LUX

- MÉdICA AÑO 8, NÚMERO 23, ENERO-ABRIL 2013, PP 21 -27
}

\begin{tabular}{|c|}
\hline Abstract \\
\hline $\begin{array}{l}\text { The depression has an approximate prevalence of } 8 \% \text { in the } \\
\text { general population. Depressive disorders are more common } \\
\text { in the female population and they have been linked to va- } \\
\text { rious vital events, including pregnancy, childbirth and puerpe- } \\
\text { rium. The objective of this study was to know the frequency } \\
\text { of depression postpartum in patients treated at the Women } \\
\text { Hospital of Aguascalientes. A cross-sectional, prospective and } \\
\text { descriptive study was conducted, patients who were enrolled in } \\
\text { the late postnatal period and that were cited in the external } \\
\text { consultation, between October I } 5 \text { and November } 16,2012 \\
\text { were included, through sampling by convenience. Edinburgh's } \\
\text { postpartum depression scale was used. I } 44 \text { Patients were } \\
\text { studied. We found a prevalence of } 21.5 \% \text {, which represents a } \\
\text { frequency higher than reported by other authors. These results } \\
\text { lead us to continue with this line of research. LUX MÉDICA AÑ } 8 \text {, } \\
\text { NúMERO 23, ENERO-ABRIL 20I3, PP } 21-27\end{array}$ \\
\hline
\end{tabular}

\section{Abstract} rious vital events, including pregnancy, childbirth and puerpeium. The objective of this study was to know the frequency postpartum in patients treated at the Women the late postnatal period and that were cited in the external consultation, between October 15 and November 16, 2012 were included, through sampling by convenience. Edinburgh's postpartum depression scale was used. 144 Patients were frequency higher than reported by other authors. These results lead us to continue with this line of research. LUX MÉDICA AÑO 8, NÚMERO 23, ENERO-ABRIL 2013, PP $21-27$

Palabras clave: puerperio tardío, depresión postparto.

\section{Introducción}

Los trastornos afectivos constituyen, en todo el mundo, la segunda alteración más frecuente entre las enfermedades mentales. ${ }^{1}$ La Organización Mundial de la Salud (OMS) considera que para el año 2020 la depresión será la primera causa de incapacidad laboral y la tercera causa de morbilidad, después de las enfermedades coronarias y de los accidentes de tránsito. Se estima que actualmente existen 150 millones de personas con depresión. ${ }^{2-3}$

* Estudiantes del noveno semestre de Medicina de la Universidad Autónoma de Aguascalientes

** Profesora investigadora del Departamento de Gíneco-Obstetricia y Pediatría del Centro de Ciencias de la Salud de la Universidad Autónoma de Aguascalientes

Fecha de recibido: 10 de enero 2013

Fecha de aceptación: 30 de enero 2013

Correspondencia: Dra Ma del Carmen Terrones Saldívar, Departamento de Gíneco-Obstetricia y Pediatría del Centro de Ciencias de la Salud, de la Universidad Autónoma de Aguascalientes. Avenida Universidad \# 940, Edificio 107, planta alta, Ciudad Universitaria. CP 20131. Aguascalientes, Ags., México Teléfono 01 (449) 9108443 Correo electrónico mcterron@correouaa.mx 
Los trastornos depresivos son más frecuentes en la población femenina y han sido asociados con diferentes eventos vitales, incluido el parto. ${ }^{4-6}$ Durante el embarazo y el puerperio suceden una serie de cambios bioquímicos, psicológicos y sociales que ocasionan mayor vulnerabilidad para la aparición de trastornos en la esfera psíquica de la mujer. ${ }^{7}$

Resulta difícil determinar la prevalencia real de la depresión posparto. La frecuencia de la depresión postparto varía sustancialmente entre los países y fluctúa entre 6.1 y $20.7 \% .^{8}$ En la literatura nacional, éste es un fenómeno que padecen entre un $15 \%$ y hasta un $32.6 \%$ de las mujeres en periodo postparto. ${ }^{8-9}$

La presentación clínica de la depresión postparto es como el de otros trastornos depresivos graves, con síntomas como disminución en el placer, cambio marcado en el apetito y el sueño, agitación o enlentecimiento psicomotor, fatiga, sentimientos de inutilidad o culpa inapropiada, disminución de la concentración, llanto frecuente, irritabilidad, rechazo hacia el recién nacido o recelo a asumir su cuidad y, en los casos graves, pensamientos recurrentes de muerte o suicidio. ${ }^{6,10-12}$ Según el Manual Diagnóstico y Estadístico de los Trastornos Mentales se puede detectar en las primeras cuatro semanas posteriores al parto. ${ }^{10}$

Llama la atención el hecho de que la depresión posparto sea habitualmente subdiagnosticada, pese a que su prevalencia parece superar la correspondiente a otras patologías obstétricas tales como preeclampsia, parto prematuro y diabetes gestacional, entre otras muchas entidades. ${ }^{13}$

La identificación oportuna y la intervención multidisciplinaria temprana de este padecimiento tienen como ventaja principal no sólo disminuir el tiempo del padecimiento, sino sobre todo reducir el efecto en el lactante menor por situaciones de desapego emocional, descuido en los cuidados básicos, abandono de la lactancia y, en algunos casos, maltrato abierto, eventos que dejan al menor muy susceptible ante los estresantes psicosociales. ${ }^{9,14-15}$

Este estudio fue realizado para determinar la prevalencia de depresión postparto en mujeres atendidas en el Hospital de la Mujer de Aguascalientes.

\section{| | | | | | | | | | | | | | | | | | | | | | | | | | | | | | | | | | | | | | | | | | | | | | | | | | | | | | | | | | | | | | | | | | | | | | | | | | | | | | | | | | | | | | | | | | | | | | | | | | ||}

\section{Material y métodos}

Realizamos un estudio descriptivo, prospectivo y transversal en el que se entrevistó a todas las pacientes que acudieron a la consulta de puerperio del Hospital de la Mujer de Aguascalientes entre el 15 de octubre y el 16 de noviembre del 2012. Todas las pacientes cursaban el puerperio tardío ( 2 a 4 semanas posparto)
Las pacientes estudiadas fueron invitadas a la cita de puerperio en el momento del alta de su evento obstétrico (parto o cesárea) y se les asignaron citas 2 a 4 semanas después de esa fecha. Los criterios de inclusión fueron: estar cursando el puerperio tardío, haber obtenido un producto vivo y que hubieran sido atendidas en el Hospital de la Mujer de Aguascalientes. 
Antes de comenzar la entrevista, a todas las pacientes que acudían a la cita se les pidió completar la prueba autoadministrada de la versión en español validada de la Escala de Depresión Postparto de Edimburgo (EPDS) $^{16-18}$, la cual consta de 10 ítems con cuatro posibles alternativas de respuesta de acuerdo a la severidad de los síntomas durante la última semana, puntuados de 0 a 3 con un rango total de 0 a 30 (Anexo 1). Fue diseñada por Cox y colaboradores en 1987 en Edimburgo, específicamente para detectar estados depresivos en el período postnatal. La escala ha demostrado ser de especial utilidad tanto en la clínica como en la investigación, habiéndose probado su eficacia para detectar estados depresivos en ámbitos hospitalarios y de atención primaria. ${ }^{16-19}$

Se usaron calificaciones de corte de 11 para obtener óptimos niveles de sensibilidad y especificidad para determinar si la paciente se encuentra en riesgo o no de padecer depresión postparto, en mujeres con menos de cuatro semanas postparto la calificación de corte de 11 tiene una sensibilidad de $75 \%$ y una especificidad de $93 \%$. $^{8}$
Durante la entrevista se abordó a la paciente sobre su estado de salud y se utilizó un cuestionario estandarizado (Anexo 2) para obtener datos sociodemográficos, clínicos y psicosociales. Las variables sociodemográficas analizadas incluyeron edad, vivienda rural o urbana, ocupación, religión, estado civil, edad al contraer matrimonio o al unirse en pareja, tiempo de vivir en pareja, relación con su cónyuge y escolaridad. Los aspectos clínicos analizados fueron número de semanas posparto, tipo de evento obstétrico, peso, estado de salud, sexo deseado y sexo real del recién nacido, así como la presencia de lactancia actual. Los datos psicosociales incluyeron presencia de alcoholismo, tabaquismo, farmacodependencia, depresión, ansiedad y estrés durante o después del embarazo, antecedente de depresión alguna vez en la vida. Los datos se concentraron en una base de datos y para reportar los resultados se utilizaron medidas de tendencia central. En caso necesario se utilizó la tStudent, con un nivel de significancia de $\mathrm{p}<0.05$

\section{| | | | | | | | | | | | | | | | | | | | | | | | | | | | | | | | | | | | | | | | | | | | | | | | | | | | | | | | | | | | | | | | | | | | | | | | | | | | | | | | | | | | | | | | | | | | | | | | || |}

\section{Resultados}

Se estudiaron un total de 144 mujeres en etapa de puerperio tardío. El promedio de edad fue de $24.45 \pm 6.48$ años (rango: 15 39 años $)$. El $47.2 \%(n=68)$ de las mujeres se ubicó entre los 20 y 29 años de edad. La mayoría de las pacientes residía en un domicilio urbano $(n=101,70.13 \%)$, se dedicaban al hogar $(n=117,81.25 \%)$, practicaban la religión católica $(n=137,95.13 \%)$ y tenían un nivel educativo máximo de secundaria incompleta $(n=66,45.83 \%)$.

En cuanto a las características de pareja, se obtuvo que el $35.41 \%(n=51)$ de las mujeres eran casadas, $45.83 \%(n=66)$ vivían en unión libre con su pareja, $16.6 \%$ $(n=24)$ solteras y $2.08 \%(n=3)$ divorciadas. El $41.66 \%(n=60)$ de las mujeres te- nía menos de 20 años de edad al casarse o unirse con su pareja, $36.8 \%(n=53)$ entre 20 y 30 años y el $4.16 \%(n=6)$ más de 30 años. El $43.05 \%(n=62)$ de las mujeres tenía menos de 3 años de tiempo de vivir en pareja y el $39.58 \%(n=57)$ más de 3 años. El $71.52 \%(n=103)$ de las mujeres dijo tener una relación satisfactoria con su pareja, mientras que el $28.48 \% \quad(n=41)$ dijo tener una relación no satisfactoria.

Los datos clínicos muestran que el $49.3 \%$ $(n=71)$ de las pacientes eran primigestas y el $50.7 \%(n=73)$ eran multigestas. Del total de las pacientes entrevistadas, la gran mayoría tuvo un recién nacido con peso normal ( $n=130,90.27 \%$ ), en buen estado de salud $(n=135,93.75 \%)$ y se encontraba lactando al momento de la entrevista 
$(n=130,90.27 \%)$. El $27.08 \%(n=39)$ de las pacientes no tuvo un recién nacido del mismo sexo del que lo deseaba. Dentro de las pacientes primigestas, el $66.19 \%(n=47)$ tuvo un parto vaginal y el resto fue sometido a cesárea $(n=24,33.81 \%)$. El $42.5 \%$ $(n=31)$ de las pacientes multigestas había tenido dos embarazos y $42(57.5 \%)$ tenían tres o más. Además, dentro de este grupo, veinte $(27.4 \%)$ pacientes multigestas habían tenido por lo menos un aborto a lo largo de su vida.

\section{Tabla |}

\section{Características sociodemográficas y prevalencia de depresión en las mujeres estudiadas}

\begin{tabular}{|c|c|c|c|c|c|c|c|c|c|}
\hline \multirow{3}{*}{$\begin{array}{l}\text { CARACTERÍSTICA } \\
\text { EDAD (años) }\end{array}$} & \multirow{3}{*}{$\begin{array}{c}\text { MUJERES } \\
\text { ESTUDIADAS }\end{array}$} & \multicolumn{3}{|c|}{ PREVALENCIA } & \multirow{3}{*}{$\begin{array}{l}\text { CARACTERÍSTICA } \\
\text { ESTADO CIVIL }\end{array}$} & \multirow{3}{*}{$\begin{array}{c}\text { MUJERES } \\
\text { ESTUDIADAS } \\
\end{array}$} & \multicolumn{3}{|c|}{ PREVALENCIA } \\
\hline & & \multicolumn{2}{|c|}{ C/DEPRESIÓN } & \multirow{2}{*}{\begin{tabular}{|c|} 
GENERAL \\
$\%$ \\
\end{tabular}} & & & \multicolumn{2}{|c|}{ C/DEPRESIÓN } & \multirow{2}{*}{$\frac{\text { GENERAL }}{\%}$} \\
\hline & & $\mathrm{n}$ & $\%$ & & & & $n$ & $\%$ & \\
\hline Menos de 20 & 45 & 7 & 22,58 & 4,86 & Unión Libre & 66 & 11 & 35,48 & 7,64 \\
\hline $20-29$ & 68 & 17 & 54,84 & 11,81 & Soltera & 24 & 6 & 19,35 & 4,17 \\
\hline 30 o más & 31 & 7 & 22,58 & 4,86 & Casada & 51 & 12 & 38,71 & 8,33 \\
\hline DOMICILIO & & & & & Divorciada & 3 & 2 & 6,45 & 1,39 \\
\hline Rural & 43 & 9 & 29,03 & 6,25 & ESCOLARIDAD & & & & \\
\hline Urbano & 101 & 22 & 70,97 & 15,28 & Primaria incom. & 23 & 2 & 6,45 & 1,39 \\
\hline OCUPACIÓN & & & & & Primaria comp. & 12 & 8 & 25,81 & 5,56 \\
\hline Trabajadora & 27 & 10 & 32,26 & 6,94 & Secundaria incomp. & 34 & 12 & 38,71 & 8,33 \\
\hline Ama de casa & 117 & 21 & 67,74 & 14,58 & Secundaria comp. & 37 & 4 & 12,90 & 2,78 \\
\hline RELIGIÓN & & & & & Bachillerato & 30 & 3 & 9,68 & 2,08 \\
\hline Católica & 137 & 27 & 87,10 & 18,75 & Licenciatura & 8 & 2 & 6,45 & 1,39 \\
\hline Otras & 7 & 4 & 12,90 & 2,78 & TOTAL & 144 & 31 & 100,00 & 21,53 \\
\hline
\end{tabular}

De las 144 mujeres estudiadas, 31 mostraron datos sugestivos de depresión. La prevalencia general de depresión postparto (EPDS $\geq 11$ ) fue de $21.52 \%$. Del total de pacientes con depresión postparto, 16 $(51.6 \%)$ eran primigestas y 15 multigestas (48.4\%).

Dentro de las 15 pacientes multigestas con depresión postparto, predominaron las mujeres con una edad entre los 20 y 29 años $(n=8,53.3 \%)$, con una vivienda urbana $(n=9,60 \%)$, amas de casa $(n=14,93.3 \%)$, con una escolaridad máxima de secundaria incompleta $(n=12,80 \%)$, y con una pareja estable $(n=13,86.7 \%)$, tienen un promedio de vivir con su pareja de 6 años. Las pacientes solteras y con depresión postparto fueron un bajo porcentaje $(n=2,13.3 \%)$. Los antecedentes de depresión alguna vez $(n=11,73.3 \%)$ o en el último embarazo $(n=11,73.3 \%)$, así como de ansiedad $(n=12,80 \%)$ o estrés $(n=13,86.7 \%)$ durante el embarazo fueron frecuentes en las pacientes con depresión postparto.

En las pacientes primigestas con depresión postnatal $(n=16)$, predominaron las mujeres con una edad entre los 20 y 29 años $(n=9,56.25 \%)$, con una vivienda urbana $(n=13,81.25 \%)$, trabajadoras $(n=9$, $56.25 \%$ ), con una escolaridad máxima de secundaria $(n=10,62.5 \%)$, y sin una pareja estable $(n=12,68.75 \%)$. Las pacientes con pareja estable y con depresión postparto $(n=5,31.25 \%)$ tuvieron un promedio de vivir en matrimonio de 4.6 años. Los antecedentes de depresión alguna vez $(n=10$, $62.5 \%)$ o en el último embarazo $(n=10$, $62.5 \%)$, así como de ansiedad $(n=10$, $62.5 \%)$ o estrés $(n=10,62.5 \%)$ durante el embarazo fueron frecuentes en las pacientes con depresión postparto. 
El antecedente de ansiedad y estrés durante el embarazo, fue una condición encontrada en 22 de las 31 pacientes con depresión postparto (70.9\%), al comparar esta condición por paridad, no encontramos diferencias estadísticamente significativas $(p=0.28)$

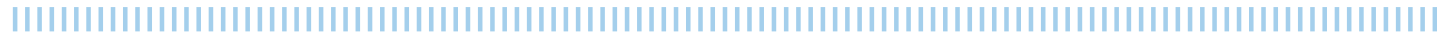

\section{Discusión}

De las 144 pacientes estudiadas, encontramos una prevalencia de $21.5 \%$ de depresión postnatal en mujeres del Hospital de la Mujer de Aguascalientes. Esta prevalencia es una de las más altas informadas en la literatura, es superior a la reportada para mujeres de Europa y Norteamérica (8 a $12 \%)$, China (11\%) y África (13.2 a 16 $\%)$. La frecuencia encontrada de depresión postparto es similar a la reportada por el Instituto Nacional de Perinatología en 2001 (21.7) y para América del sur (20.7\%) y es menor a la reportada en Sudáfrica (34.7 $\%)$, algunos países de Asía (40\%) y algunos estados de nuestro país, tales como Durango (32.6\%). ${ }^{8}$, 20-22 La elevada prevalencia de depresión postparto en pacientes del Hospital de la Mujer, puede deberse a las diferencias en el tipo y frecuencia de los factores de riesgo para la depresión entre los países.

La corta edad materna se ha asociado con depresión postparto en diversas investigaciones, ${ }^{23}$ en nuestro estudio encontramos una mayor frecuencia de depresión postparto en las mujeres multigestas de entre 20-29 años de edad $(n=17,54.8 \%)$.

El estado civil de soltera se ha asociado con la depresión en estudios anteriores. ${ }^{24}$ Sin embargo en nuestro estudio el $58.06 \%$ $(n=18)$ de las mujeres con depresión postparto contaba con una pareja estable.

La depresión postparto fue más frecuente en aquellas mujeres cuya educación era insuficiente. Veintidós casos de depresión postparto fueron reportados en mujeres con educación básica solamente (máximo grado de estudios fue secundaria completa), lo que representa un $70.96 \%$ del total de 31 casos de depresión postparto encon- trados durante la investigación. Lo anterior corrobora lo expuesto en la literatura internacional en donde se relaciona el bajo nivel educativo con una mayor prevalencia de trastornos afectivos del postparto. ${ }^{25}$

También se encontró que las mujeres con más de tres años de vivir con su pareja mostraron una frecuencia mayor de depresión postparto $(n=13,41.93 \%)$ que las mujeres con una relación más corta $(n=10$, $32.2 \%$ ). Esta circunstancia no es esperada si consideramos que las mujeres con varios años de relación de pareja pudieran sentir mayor seguridad y estabilidad emocional que las que tienen pocos años. Sin embargo, las familias con más de tres años de duración pueden tener más necesidades financieras y quizás más conflictos que aquellas con una duración menor, situación que nos puede explicar el resultado anterior. ${ }^{8}$ Esta característica ha sido escasamente explorada en estudios epidemiológicos y merece ser considerada en nuevas investigaciones.

La lactancia ha sido mencionada como factor protector para varios trastornos afectivos del puerperio, incluyendo la depresión. ${ }^{21}$ En nuestro estudio encontramos que la mayor parte de las pacientes con depresión postnatal estaban lactando $(n=26$, $83.87 \%$ ). Es importante mencionar que el Hospital de la Mujer de Aguascalientes es reconocido como Hospital Amigo de la madre y el niño de la OMS y UNICEF, programa para implementar prácticas que protejan, promuevan y apoyen la lactancia materna. ${ }^{26}$

Se ha reportado que en las mujeres multigestas, existe una probabilidad mayor de depresión postparto. ${ }^{5,27}$ Sin embargo otros autores no han encontrado una relevancia entre el padecimiento y la paridad de las 
mujeres evaluadas. ${ }^{6,14,28}$ En nuestro estudio encontramos que de las mujeres con depresión postparto, aproximadamente una de cada tres pacientes $(n=10,32.25 \%)$ son mujeres con tres o más embarazos. No encontramos diferencia significativa entre la prevalencia de depresión postnatal en pacientes multígestas $(n=15,48.38 \%)$ y primigestas $(n=16,51.61 \%)$.

La literatura indica que cerca de $50 \%$ de los casos de depresión postparto son consecuencia de estados depresivos que ya se habían presentado en el embarazo o incluso antes de éste, las mujeres que sufren este cuadro tienen un mayor riesgo de sufrir episodios futuros de depresión, con o sin relación a un parto, ${ }^{28,29}$ este porcentaje se reduce a menos del $2 \%$ si se efectúa un tratamiento psicoterapéutico adecuado durante dicho periodo. ${ }^{30}$ La depresión prenatal es uno de los predictores más fuertes de depresión postparto. ${ }^{19}$ En nuestro estudio, el $67.74 \%(n=21)$ y el $70.9 \%(n=22)$ de las mujeres con depresión postparto, refirieron haber padecido depresión y ansiedad alguna vez o durante el embarazo, respectivamente.

A pesar de que el número de pacientes que estudiamos corresponde a una muestra pequeña, los resultados de este trabajo nos ponen en alerta ante un problema que no se ha identificado con anterioridad en nuestro estado.

\section{Conclusiones}

La prevalencia de depresión postnatal en el Hospital de la Mujer de Aguascalientes, México, es alta en comparación con las señaladas en otros estudios; diversos factores sociodemográficos, clínicos y psicosociales contribuyeron para la depresión, estos antecedentes podrían dar cuenta de la vulnerabilidad biológica y psicosocial presente en estas pacientes. La baja escolaridad y el antecedente de algún trastorno afectivo como depresión, ansiedad o estrés fueron situaciones frecuentes en las mujeres en las que se encontró depresión postparto. La capacitación del equipo de salud se hace, entonces, imprescindible para mejorar la salud mental familiar $y$, en especial, la del binomio madre-hijo.

\section{Bibliografía}

1. Latorre JF, Contreras LM, García S, Arteaga J (2006). La depresión postparto en madres adolescentes de Bucaramanga, Colombia. Revista Colombiana de Obstetricia y Ginecología; Vol. 57, No. 3: 156-162.

2. Trejo-Lucero H, Torres J, Medina MA, Briones JC, Meneses J (2009). Depresión en adolescentes embarazadas que acuden a un hospital perinatal. Rev Mex Pediatr; 76 (1): 9-12.

3. World Health Organization (2010) Mental health and development: Targeting people with mental health conditions as a vulnerable group. WHO Press.

4. Latorre JF, Contreras LM, Herrán OF (2006). Depresión posparto en una ciudad colombiana. Factores de riesgo. Aten Primaria; 37(6):332-8.
5. Bjerke $S E$, Vangen $S$, Nordhagen $R$, Ytterdahl $T$, Magnus P, Stray-Pedersen B (2008). Postpartum depression among Pakistani women in Norway: Prevalence and risk factors. The Journal of Maternal-Fetal and Neonatal Medicine; 21 (12): 889-894.

6. Urdaneta J, Rivera A, García J, Guerra M, Baabel N, Contreras A (2010). Prevalencia de depresión posparto en primigestas y multíparas valoradas por la escala de Edimburgo. Rev Chil Obstet Ginecol; 75(5): 312-320.

7. Field T (2010). Postpartum Depression effects on early interactions, parenting, and safety practices: a review. Infant Behav Dev. February; 33 (1):1.

8. Alvarado $C$, Sifuentes $A$, Estrada $S$, Salas $C$, Hernández AB, Ortiz SG, García CR, Torres A, Sandoval F (2010).Prevalencia de depresión posnatal en mujeres 
atendidas en hospitales públicos de Durango,México. Gac Méd Méx Vol. 146 No. 1.

9. Arranz LC, Aguirre W, Ruiz J, Gaviño S, Cervantes JF, Carsi E, Camacho M, Ochoa MG (2008). Enfoque multidisciplinario en la depresión posparto. Ginecol Obstet Mex; 76(6):341-8.

10. Asociación Americana de Psiquiatría (2002) DSMIV-TR; Manual Diagnóstico y estadístico de los trastornos mentales. American Psychiatric Association, Whashington, DC.

11. Rojas G, Fritsch R, Solís J, González M, Guajardo V, Araya R (2006). Calidad de vida de mujeres deprimidas en el postparto. Rev Méd Chile; 134:713-720.

12. Gjerdingen DK, Yawn BP (2007). Postpartum depression screening: importance, methods, barriers, and recommendations for practice. J Am Board Fam Med May-June; Vol. 20 No. 3.

13. Moreno A, Domingues L, Fran(a PS (2004). Depresión postparto: prevalencia de test rastreo positivo en puérperas del Hospital Universitario de Brasilia, Brasil. Rev Chil Obstet Ginecol; 69 (3): 209-213.

14. Morris-Rush JK, Comerford M, Bernstein P (2003). Screening for postpartum depression in an inner-city population. Am J Obstet Gynecol; 188:1217-1219.

15. Barra F, Barra L, Solís J (2009). Depresión postparto. Hacia un tratamiento integral. Actualizaciones en Psicoterapia Integrativa; Vol. I, 77-88.

16. Cox JL, Holden JM, Sagovsky R (1987). Detection of postnatal depression. Development on the 10-item Edinburgh Postnatal Depression Scale. BJP June; 150:782-6.

17. Castañón C, Pinto J (2008). Mejorando la pesquisa de depresión posparto a través de un instrumento de tamizaje, la escala de depresión posparto de Edimburgo. Rev Méd Chile; 136: 851-858.

18. Gibson J, McKenzie K, Shakespeare J, Price J, Gray R (2009). A systematic review of studies validating the Edinburgh Postnatal Depression Scale in antepartum and postpartum women. Acta Psychiatr Scand; 119: 350-364.

19. Sylvén, SM (2012). Biological and Psychosocial Aspects of Postpartum Depression. Acta Universitatis Upsaliensis. Digital Comprehensive Summaries of Uppsala Dissertations from the Faculty of Medicine
751. 71 pp. Uppsala, Sweden. ISBN 978-91-5548302-9.

20. Álvarez A, Ponce ER, Irigoyen A (2008). Frecuencia de depresión posparto en pacientes de dos clínicas de medicina familiar en México. Archivos en Medicina Familiar; Vol. 9 (4) 133-136.

21. Watkins S, Meltzer S, Zolnoun D, Stuebe A (2011). Early breastfeeding experience and postpartum depression. Obstet Gynecol; 118:214-21.

22. Hedge S, Latha KS, Bhat S, Sharma PSVN, Kamath A, Shetty A (2012). Postpartum Depression: prevalence and associated factors among women in India. J Womens Health, Issues \& Care, 1:1:1-7.

23. Wolff C, Valenzuela P, Esteffan K, Zapata D (2009). Depresión posparto en el embarazo adolescente: análisis del problema y sus consecuencias. Rev Chil Obstet Ginecol; 74 (3): 151-158.

24. Robetson E, Celasun N and Stewart DE (2003). Risk factors for postpartum depression. In Stewart DE, Robertson E, Dennis CL, Grace SL \& Wallington T (2003). Postpartum depression: Literature review of risk factors and interventions.

25. Reck C, Struben K, Backenstrass $M$, Stefenelli U, Reining K, Fuchs T (2008). Prevalence, onset and comorbidity of postpartum anxiety and depressive disorders. Acta Psychiatr Scand; 118:459-68

26. UNICEF Venezuela, Hacia la promoción y rescate de la lactancia materna. 2da edición 2005.

27. Rojas G, Fritsch R, Guajardo V, Rojas F, Barroilhet $S$, Jadresic E (2010). Caracterización de madres deprimidas en el postparto. Rev Med Chile; 138:536542.

28. Orejarena SJ (2004). Trastornos afectivos posparto. MedUNAB; 7:134-9.

29. Cooper P, Murray L, Halligan S (2010). Treatment of postpartum depression. Encyclopedia on Early Childhood Development. Center of Excellence for Early Childhood Development.

30. Martínez P, Romano WK (2009). Depresión en adolescentes embarazadas. Enseñanza e Investigación en Psicología; 14(2): 261-274. Disponible en:

http://redalyc.uaemex.mx/src/inicio/ArtPdfRed. jsp?iCve $=29211992004$

Parte de este trabajo fue premiado en el Primer Encuentro de Jóvenes Investigadores del CONACYT, realizado en la Universidad Autónoma de Aguascalientes en noviembre del 2012. 